Article

\title{
In Vitro and in Vivo Evaluation of the Antioxidant and Prooxidant Activity of Phenolic Compounds Obtained from Grape (Vitis vinifera) Pomace
}

\section{Milena Cotoras ${ }^{1, *}$, Herman Vivanco ${ }^{1}$, Ricardo Melo ${ }^{1}$, María Aguirre ${ }^{2}$, Evelyn Silva ${ }^{1}$ and Leonora Mendoza ${ }^{1, *}$}

1 Laboratorio de Micología, Facultad de Química y Biología, Universidad de Santiago de Chile, Alameda 3363, Estación Central, Santiago 916000, Chile;

E-Mails: herman.vivancov@usach.cl (H.V.); ricardo.melo@usach.cl (R.M.); evelyn.silva@usach.cl (E.S.)

2 Laboratorio de Electroquímica, Facultad de Química y Biología, Universidad de Santiago de Chile, Alameda 3363, Estación Central, Santiago 916000, Chile; E-Mail: maria.aguirre@usach.cl

* Authors to whom correspondence should be addressed; E-Mails: milena.cotoras@usach.cl (M.C.); Leonora.mendoza@usach.cl (L.M.); Tel.: +56-2-2718-1094 (M.C.); +56-2-2718-1062 (L.M.); Fax: +56-2-2681-2108 (M.C. \& L.M.).

External Editor: Derek J. McPhee

Received: 6 November 2014; in revised form: 8 December 2014 / Accepted: 9 December 2014 / Published: 16 December 2014

\begin{abstract}
The antioxidant and/or prooxidant ability of extracts obtained from wine waste were analyzed using in vitro and in vivo assays. Cyclic voltammetry was used as the in vitro assay to determine the antioxidant and/or prooxidant properties and, the in vivo effect on mycelial growth of the fungus Botrytis cinerea was evaluated. In addition, the prooxidant activity was evaluated by intracellular oxidation of compound 2,7-dichlorodihydrofluorescein diacetate (DCFH-DA) in B. cinerea. The extracts used in this study were obtained from grape pomace of Cabernet Sauvignon, Carménère and Syrah varieties from the Misiones de Rengo Vineyard by simple extraction, using methanol/HCl $1 \%(\mathrm{v} / \mathrm{v})$, ethanol $70 \%(\mathrm{v} / \mathrm{v})$, or Soxhlet extraction. According to the results obtained, gallic acid was the most represented phenolic compound independent of grape variety and extraction method. In addition, vanillic acid; protocatechuic acid, syringic acid, quercetin and kaempferol were found in the extracts. From this study it was possible concluded that, depending of the method of extraction of the grape residues and the grape variety
\end{abstract}


(Cabernet Sauvignon, Carménère and Syrah), the extracts showed antioxidant and/or prooxidant activity. However, no correlation can be established between the anodic oxidation potentials of the extracts and their effect on the fungus $B$. cinerea.

Keywords: grape pomace; phenolic compounds; antioxidant and prooxidant activity

\section{Introduction}

Wine industry produces two types of wastes, wastewater and organic solid wastes generating a noticeable environmental impact [1]. The main organic wastes produced in modern wine industries include grape pomace (62\%), lees (14\%), stalk (12\%) and dewatered sludge (12\%). Currently, only a minimal amount of these residues are recycled [2].

Grape pomace consists mainly of skin residues, broken cells with pulp remains, stalks, and seeds [1]. Its composition varies depending on grape variety climate, culture localization and technology of vinification [3]. In addition, it has been described that grape pomace contains a great amount of anthocyanins, catechins, flavonoles, alcohols, stilbenes and phenolic compounds. These last compounds have pharmaceutical and agronomical applications since antioxidant and free radical scavenging, anticancer, antifungal and antibacterial activities have been reported. Among these biological properties, the best known and most widely studied is the antioxidant effect, which has been the focus of a large amount of analysis, mostly of clinical and nutritional nature [4].

In particular, it has been described that citrus peel [5], apple and grape pomace [6,7] contain several phenolic compounds. Among them catechins (catechin, epicatechin), flavonols (quercetin, kaempferol, myricetin), benzoic acids (gallic, protocatechuic, 4-hydroxybenzoic, syringic, gentisic) and cinnamic acids (p-coumaric) are representative [8]. Recently, Mendoza et al. [9] compared different extraction techniques to obtain grape pomace extracts with antifungal activity. These authors reported that quercetin was a phenolic compound most representative from a mixture of Chilean grape varieties (Cabernet Sauvignon, Carmènere and Syrah). However, kaempferol, vanillic and syringic acid were also identified. From this study, it was possible conclude that the grape pomace can be considered as a good low cost source to obtain extracts with antifungal activity against the phytophatogenic fungus Botrytis cinerea. This fungus, the causal agent of gray mould, is a broad host pathogen that derives its sustenance from dead or necrotic plants. In particular, the infections produced by Botrytis result in great economic losses, not only during growth but also during storage and transport. In particular, grapes are one of the commodities most affected by Botrytis infection [10].

Characterizations of phenolic compounds have pointed them out as powerful in vitro antioxidants, even more potent than Vitamins $\mathrm{C}$ and $\mathrm{E}$ and the carotenoids [11,12]. However, it was also found that phenolic antioxidants behave like prooxidants under the conditions that favor their autoxidation, for example, at high $\mathrm{pH}$ with high concentrations of transition metal ions and oxygen molecules present. Small phenolics that are easily oxidized, such as quercetin, gallic acid, possess prooxidant activity; while high molecular weight phenolics, such as condensed and hydrolysable tannins, have little or no prooxidant activity [13]. Supporting this observation, it has been described that quercetin and caffeic acid take part in redox reactions in which they can act as either antioxidants (electron donors) or 
prooxidants (electron acceptors), depending on their environment. In addition, Fukumoto and Mazza noted dual antioxidant and prooxidant activities for a variety of plant-derived polyphenols, including gallic acid, protocatechuic acid, syringic acid, vanillic acid, ellagic acid, caffeic acid, coumaric acid, chlorogenic acid, ferulic acid, myricetin, quercetin, rutin, kaempferol, (+)-catechin, (-)-epicatechin, delphinidin, and malvidin [14]. Therefore, the prooxidant activity of phenolic compounds would explain the antifungal effect previously reported [9]. In Candida spp., it has been shown that the phenolic compound curcumin increased the reactive oxygen species level and induced early apoptosis [15]. In Rhyzopus spp and in Aspergillus the fluidity of the membrane was disrupted by interaction of phenolic compounds with ergosterol [16,17]. In $B$. cinerea the 5,7-dihydroxy-3,8-dimethoxyflavone partially affected conidial germination, reduced oxygen consumption and, affected the plasma membrane integrity [18].

Hence, this work aimed to the achieve the following: (i) analyze phenolic compounds obtained from different pomace grape varieties by different extraction methods; (ii) determine the in vitro antioxidant/prooxidant activity from each extract by voltammetry cyclic and (iii) evaluate the in vivo prooxidant activity using $B$. cinerea as model organism.

\section{Results and Discussion}

\subsection{Analysis of Antioxidant and Prooxidant Activity and Phenolic Compound Composition from} Different Pomace Grape Varieties

In this work, whole or ground grape pomace samples from the varieties Cabernet Sauvignon, Carménère and Syrah and two-extraction methods (solid-liquid extraction and Soxhlet) were tested to obtain polyphenol-enriched extracts (Table 1). Later, each extract was submitted to liquid-liquid extraction using different solvent as a hexane, methanol, chloroform and ethyl acetate. The higher extraction yield, in all grape varieties (Cabernet Sauvignon, Carménère and Syrah) was obtained by means of simple extraction method using methanol/HCl $1 \%(\mathrm{v} / \mathrm{v})$. These results are concordant with the described by Castañeda-Ovando et al. [19]. According to the type or sample of pomace grape, the highest yield (30\%) was obtained from ground pomace Cabernet Sauvignon by simple extraction instead of Soxhlet extraction which only produced a maximum of $12 \%$ yield in all strains (data not shown). On the other hand, the total phenol concentration in the different fractions varied among 300 and $1000 \mathrm{mg} / \mathrm{mL}$. There was no correlation between the extraction method or the grape pomace variety and the total phenol concentration (data not shown). In addition, phenolic compounds in the different fractions were identified by HPLC. The results are also shown in Table 1.

It has been widely reported that the extraction method can affect the phenolic profile of the resulting extracts [4]. In addition, it has been also described that extracts obtained by liquid extraction from whole substrates showed lower yields than from ground substrates [9]. Based on the previous results, the presence of different phenolic compounds in different fractions was not a surprise. As might be expected, several phenolic compounds were identified. Among them, gallic acid was the most represented. This phenolic acid was present independent of grape variety and extraction method. It has been reported that gallic acid is one of the major phenolic compounds founded in grape seeds and skins together with catechin and epicatechin [20]. Another highly represented phenolic compound was 
vanillic acid. However, this compound was lacking in the grape varieties treated with ethanol-system 2. Interestingly, protocatechuic acid was also highly represented in the different grape varieties independent of extraction method but, like vanillic acid, it was not possible to identify this compound on the fraction extracted with ethanol-system 2. It has been described that protocatechuic acid has an antioxidant effect due to its capacity to chelate metal ions and scavenge free radicals [21]. On the other hand, 4-hydroxyphenylacetic acid was identified only in the fraction extracted with ethanol system 2. Other compounds identified were syringic acid, quercetin and kaempferol, evenly distributed across the extraction methods.

Table 1. Pomace grape variety and extraction; oxidation potentials, chemistry composition from grape pomace extracts using different extraction methods. Epa corresponds to anodic oxidation potential and script $\mathrm{a}$ and $\mathrm{b}$ represents different oxidation peaks.

\begin{tabular}{|c|c|c|c|c|c|c|}
\hline $\begin{array}{l}\text { Extraction } \\
\text { Method }\end{array}$ & Grape Variety & Fraction & Sample & $\begin{array}{l}\text { Epa } \\
(\mathbf{V})^{\text {a }}\end{array}$ & $\begin{array}{l}\text { Epa } \\
(\mathbf{V})^{b}\end{array}$ & Phenolic Composition \\
\hline \multirow{4}{*}{ Methanol/HCl } & $\begin{array}{l}\text { Cabernet } \\
\text { Sauvignon }\end{array}$ & \multirow{4}{*}{ Ethyl acetate } & Ground & 0.4 & 0.75 & $\begin{array}{l}\text { vanillic acid, syringic acid, gallic acid, kaempferol, quercetin, } \\
\text { protocatechuic }\end{array}$ \\
\hline & Carménère & & Whole & 0.4 & 0.8 & $\begin{array}{c}\text { vanillic acid, syringic acid, } \\
\text { gallic acid, elagic acid, quercetin, } \\
\text { 4-hydroxi-3,5-dimethoxibenzaldehyde }\end{array}$ \\
\hline & \multirow[t]{2}{*}{ Syrah } & & Ground & 0.35 & 0.7 & $\begin{array}{c}\text { gallic acid, p-coumaric, elagic acid, } \\
\text { quercetin, kaempferol }\end{array}$ \\
\hline & & & Whole & 0.4 & 0.8 & $\begin{array}{l}\text { gallic acid, protocatechuic, vanillic acid, } \\
\text { syringic acid, quercetin, kaempferol }\end{array}$ \\
\hline \multirow{11}{*}{$\begin{array}{c}\text { Ethanol } \\
\text { (System 1) }\end{array}$} & \multirow{4}{*}{$\begin{array}{c}\text { Cabernet } \\
\text { Sauvignon }\end{array}$} & Hexane & Whole & - & 0.6 & - \\
\hline & & & Ground & 0.35 & - & $\begin{array}{l}\text { gallic acid, vanillic acid, syringic acid, } \\
(-) \text { epicatechin, quercetin, kaempferol }\end{array}$ \\
\hline & & Chloroform & Whole & - & 0.7 & $\begin{array}{l}\text { gallic acid, protocatechuic acid, vanillic acid, syringic acid, elagic } \\
\text { acid, quercetin, kaempferol }\end{array}$ \\
\hline & & Ethyl acetate & Ground & - & 0.6 & gallic acid, $(-)$ epicatechin, quercetin \\
\hline & \multirow{3}{*}{ Carmènére } & \multirow{2}{*}{ Chloroform } & Ground & 0.3 & - & vanillic acid, syringic acid, quercetin, kaempferol \\
\hline & & & Whole & 0.41 & - & vanillic acid, syringic acid, quercetin, kaempferol \\
\hline & & Ethyl acetate & & 0.4 & - & $\begin{array}{l}\text { gallic acid, protocatechuic acid, } \\
\text { quercetin, }(-) \text { epicatechin }\end{array}$ \\
\hline & \multirow{4}{*}{ Syrah } & Chloroform & Ground & 0.38 & - & $\begin{array}{l}\text { gallic acid, vanillic acid, syringic acid, } \\
\text { quercetin, kaempferol }\end{array}$ \\
\hline & & & Whole & 0.34 & - & vanillic acid, syringic acid, quercetin, kaempferol \\
\hline & & Ethyl acetate & Ground & 0.3 & - & $\begin{array}{l}\text { gallic acid, vanillic acid, syringic acid, } \\
\text { quercetin, }(-) \text { Epicatechin }\end{array}$ \\
\hline & & & Whole & 0.35 & 0.8 & $\begin{array}{l}\text { gallic, protocatechuic acid, } \\
\text { vanillic acid, p-coumárico, Elagic acid }\end{array}$ \\
\hline \multirow{9}{*}{$\begin{array}{c}\text { Ethanol } \\
\text { (System 2) }\end{array}$} & \multirow{3}{*}{$\begin{array}{l}\text { Cabernet } \\
\text { Sauvignon }\end{array}$} & \multirow[t]{2}{*}{ Chloroform } & Ground & - & 0.58 & $\begin{array}{c}\text { syringic acid, quercetin,kaempferol, } \\
\text { 4-hydroxiphenyl acetic acid }\end{array}$ \\
\hline & & & Whole & - & 0.57 & 4-hydroxiphenil acetic acid, syringic acid, quercetin, kaempferol \\
\hline & & Ethyl acetate & Ground & 0.31 & - & gallic acid, quercetin, (-) Epicatechin \\
\hline & \multirow{3}{*}{ Carménère } & \multirow{3}{*}{ Chloroform } & Ground & 0.29 & - & gallic acid, Catequin, quercetin, $(-)$ Epicatechin \\
\hline & & & Whole & - & 0.55 & gallic acid, Catequin, quercetin, $(-)$ Epicatechin \\
\hline & & & Ground & 0.34 & - & - \\
\hline & \multirow{3}{*}{ Syrah } & Hexane & Whole & - & 0.6 & quercetin \\
\hline & & Chloroform & Ground & 0.32 & - & 4-hydroxiphenil acetic acid, syringic acid, quercetin, kaempferol \\
\hline & & Ethyl acetate & Ground & 0.33 & - & gallic acid, vanillic acid, quercetin, $(-)$ Epicatechin \\
\hline
\end{tabular}


Currently several authors have described that phenolic compounds extracted from plants materials, have antioxidative properties in various model systems and in several foods, where they are finding increasing use [22-24]. However, it has been also described that these compounds can exhibit prooxidant and cytotoxic properties under certain conditions such as the presence of metals, $\mathrm{pH}$, structural characteristics and concentration [25]. On the other hand, it has been shown that mixtures of different phenolic compounds can produce synergistic or antagonistic prooxidant effects [26]. Due to the fact a mixture of phenolic compounds is found in the grape pomace extracts, in this work, antioxidant and prooxidant activities of extracts obtained from $V$. vinifera residues were determined using different approaches.

In the first one, the antioxidant and prooxidant activity was determined in vitro by a cyclic voltammetry assay using the criteria described by Simić et al. These authors established that compounds with low oxidation potentials (anodic oxidation potential (Epa) lower than 0.45) showed antioxidant activity, whereas compounds with high Epa values $(>0.45)$ acted as prooxidants [27]. Figure 1 shows an example of a voltammogram corresponding to the chloroform extract from Syrah variety obtained by solid-liquid extraction using 70\% ethanol (v/v) (system 1), where an anodic oxidation peak is observed (Epa) at $0.38 \mathrm{~V}$.

Figure 1. Voltammogram of chloroform extract from Syrah $(350 \mathrm{ppm})$ dissolved in $\mathrm{DMF} / 0.1 \mathrm{M}$ TBAP at $10 \mathrm{mV} / \mathrm{s}$.

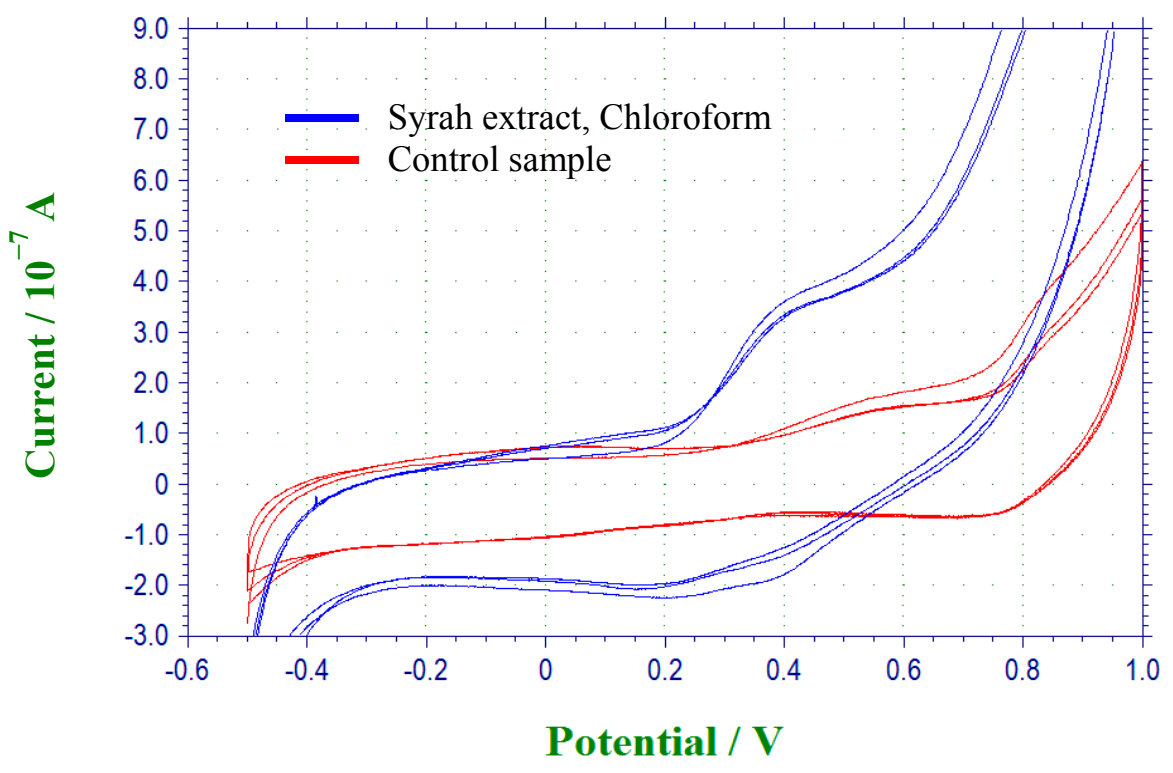

Based on this technique, the antioxidant or prooxidant ability of grape pomace extracts were obtained and some values are listed in the Table 1, where Epa corresponds to the anodic oxidation potential and the subscripts (a) and (b) represent different oxidation peaks. It is necessary to comment that only those fractions that showed cyclic voltammetry signals are represented. According to the results shown in Table 1, it is possible to observe that in the methanol extraction from grape varieties, only the ethyl acetate fraction showed signals by cyclic voltammetry. These results could be explained because this solvent (ethyl acetate) presents higher polarity than the other solvents used, allowing a better extraction of phenolic compounds that can be oxidized and reduced in that range. Therefore, these extracts would present both antioxidant and prooxidant activities. An explanation could be that, in 
general, extracts that exhibited low oxidation potential values contained easily oxidized phenolic compounds such us quercetin, kaempferol, elagic acid, and protocatechuic according to describe by Simić et al. These authors investigated the electrochemical oxidation of a number natural phenolics (salicylic acid, $m$-hydroxybenzoic acid, $p$-hydroxybenzoic acid, protocatechuic acid, $o$-coumaric acid, $m$-coumaric acid, $p$-coumaric acid, caffeic acid, quercetin and rutin) suggesting that multiple $\mathrm{OH}$ substitution and conjugation are important determinants of the free radical scavenging activity and electrochemical behavior. As we mentioned previously, compounds with low oxidation potentials showed antioxidant activity, whereas compounds with high Epa values act as prooxidants. This characteristic could explain an antagonistic interaction with phenolic compounds that present higher oxidation potential values (vanillin and syringic acid) present in some extracts simultaneously displaying antioxidant and prooxidant activity. In our case, the phenolic compounds present in the fraction treated with methanol/HCl extraction are similar those mentioned by Simić et al. [27].

On the other hand, the ethanol extractions (system 1 and system 2) showed differences in antioxidant or prooxidant activity for the different grape varieties as determined by cyclic voltammetry. System 1 presented a greater presence of fractions with only antioxidant ability than system 2 where the presence of antioxidant and prooxidant ability appears to be balanced. If grape varieties are compared, it is possible to observe that the Cabernet Sauvignon variety presented mainly a prooxidant activity, except when the extracts were obtained with chloroform where oxidant ability is present (ground sample). The activity exhibited by Cabernet Sauvignon variety could be attributed to the presence of other compounds with properties to oxidize in the range measured by cyclic voltammetry despite the fact phenolic compounds were not detected in this fraction. Besides that factors that could clarity the oxidant activity in the chloroform fraction are the presence of vanillic and syringic acids identified in these fractions. Supporting this observation, it has been described that the methylation of the 3-hydroxyl group in protocatechuic acid to form vanillic acid caused a significant reduction of the radical scavenging capacity and the introduction of another methoxy group in the 5-position to form syringic acid slightly decreased the prooxidant activity (Simić et al. [27]). Regarding the Carménère extracts, they presented antioxidant activity independent of the solvent used and prooxidant activity was founded only in a fraction treated with chloroform. With respect to the Syrah variety, all fractions showed antioxidant activity, except the hexane fraction in the system 2 that presented a prooxidant activity. These findings could be explained based on the fact that this fraction contains phenolic compounds such as catechin, quercetin, kaempferol and gallic acid for which higher levels of antioxidant activity have been reported compared with those with a single hydroxyl group [28].

\subsection{Evaluation of in Vivo Prooxidant Ability of the Extracts}

It has been widely described that phenolic compounds originating from various plant sources are important anti-inflammatory, anti-histamine, antiviral, antibacterial and even antifungal agents due to their antioxidant or prooxidant capacity [28,29]. In the case of phenolic acids, the structure of these compounds is crucial because the antioxidant activity depends on the number and position of hydroxyl groups relative to the carboxyl functional group [12]. In this work, the in vivo prooxidant ability of the extracts was analyzed using the phytopathogenic fungus $B$. cinerea as a study model. For this, a series of extracts with antioxidant and/or prooxidant activity ability determined by cyclic voltammetry assay 
were selected to evaluate the $B$. cinerea growth under their presence. The results are presented in the Table 2.

Table 2. Effect of extracts of selected Carménère, Cabernet and Syrah varieties on mycelial growth of Botrytis cinerea. Epa corresponds to anodic oxidation potential and script $\mathrm{a}$ and $\mathrm{b}$ represents different oxidation peaks.

\begin{tabular}{|c|c|c|c|c|c|c|}
\hline Extraction Solvent & Grape Variety & Fraction & Sample & $\begin{array}{c}\text { Antioxidant Epa } \\
(\mathrm{V})^{\mathrm{a}}\end{array}$ & $\begin{array}{c}\text { Prooxidant Epa } \\
(\mathrm{V})^{\mathrm{b}}\end{array}$ & $\begin{array}{c}\operatorname{ED}_{50} \pm \mathrm{SD} \\
(\mu \mathrm{g} / \mathrm{mL})\end{array}$ \\
\hline \multirow{2}{*}{ Methanol/HCl } & Carménère & \multirow{2}{*}{ Ethyl acetate } & \multirow{2}{*}{ Whole } & 0.4 & 0.8 & $52.04 \pm 3.32$ \\
\hline & Syrah & & & 0.4 & 0.8 & $50.06 \pm 3.34$ \\
\hline \multirow{3}{*}{$\begin{array}{c}\text { Ethanol 70\% } \\
\text { (System 1) }\end{array}$} & Cabernet Sauvignon & \multirow{2}{*}{ Chloroform } & Ground & 0.35 & - & $49.34 \pm 6.24$ \\
\hline & Syrah & & Whole & 0.38 & - & $50.76 \pm 2.58$ \\
\hline & Syrah & Ethyl acetate & Whole & 0.35 & 0.8 & $44.59 \pm 7.75$ \\
\hline \multirow{3}{*}{$\begin{array}{c}\text { Ethanol 70\% } \\
\text { (System 2) }\end{array}$} & Cabernet Sauvignon & Ethyl acetate & Ground & 0.31 & - & $41.97 \pm 5.23$ \\
\hline & Carménère & Chloroform & Whole & - & 0.55 & $22.81 \pm 3.28$ \\
\hline & Syrah & Ethyl acetate & Ground & 0.33 & - & $72.64 \pm 7.27$ \\
\hline
\end{tabular}

It is possible to observe that all the fractions inhibited the hyphal growth of $B$. cinerea expressed in relation to $\mathrm{IC}_{50}$ (concentration that reduced mycelial growth by $50 \%$ ). The higher antifungal activity was obtained from chloroform extract from Carménère variety extracted with $70 \%$ ethanol (system 2 ). This extract showed only an oxidation peak in the voltagramm corresponding to prooxidant activity and phenolic compounds as a gallic acid, catequin, quercetin and (-) epiquercetin were identified (data not shown). On the other hand, a lower effect was observed with the same pomace grape variety and extraction method but using ethyl acetate as a solvent. It should be noted that this fraction has a phenolic composition similar to that of the fraction with higher antifungal activity but additionally it contained vanillic acid. It is tempting to suggest that the inhibition depends on the presence of antioxidant polyphenols such as catequin, epicatequin and quercetin, which would enhance the antifungal effects. Supporting this observation, it has been described that catequin, a polyphenol very abundant in tea plants, is a strong radical scavenger and metal chelator in model chemical systems, and these effects correlate with the presence of the dihydroxy and trihydroxy groups [30]. In addition, an increasing number of studies have also demonstrated these antioxidative effects in vivo [28,29]. In contrast, the presence of vanillic acid, a phenolic compound with a high oxidation potential, unlike catequin or gallic acid [27,31] would produce less growth inhibition effect on $B$. cinerea.

To elucidate if the inhibition of $B$. cinerea mycelium growth in the presence of the extracts is due to the prooxidant activity of these components in vivo intracellular oxidation assays were performed. To analyze the intracellular oxidation, the DCFH-DA probe was used. This probe is cell permeable and is hydrolyzed by cellular esterases to the DCFH carboxylate anion, which is oxidized by reactive species to a fluorescent product, dichlorofluorescein (DCF) [32]. Fungal mycelia were incubated in presence of $40 \mathrm{ppm}$ of the extracts for $6 \mathrm{~h}$ and the oxygen reactive species (ROS) registered. As a positive control farnesol was used. Farnesol is an isoprenoid alcohol that has been described to increase ROS levels, inhibit growth and induce cell death in yeast and fungi, including $B$. cinerea, Aspergillus nidulans, Candida albicans and Saccharomyces cerevisiae [33,34]. The results are presented in the Figure 2. 
Figure 2. Detection of intracellular ROS by DCFH-DA in B. cinerea hyphae. $(\mathbf{A}, \mathbf{B})$ corresponds to positive controls, farnesol $(100 \mathrm{mM})$ and $\mathrm{H}_{2} \mathrm{O}_{2}$ (300 $\left.\mathrm{mM}\right)$ respectively; (C) negative control (methanol); (D) Carménère variety, ethyl acetate fraction (40 ppm) obtained by methanol/HCl 1\% (v/v) extraction method; (E,F) chloroform fraction $(40 \mathrm{ppm})$, strain and ethyl acetate $(40 \mathrm{ppm})$ fraction from, Carménère and Syrah varieties respectively obtained by using ethanol $70 \%$ (v/v) (system 2). All the samples were incubated for $6 \mathrm{~h}$.
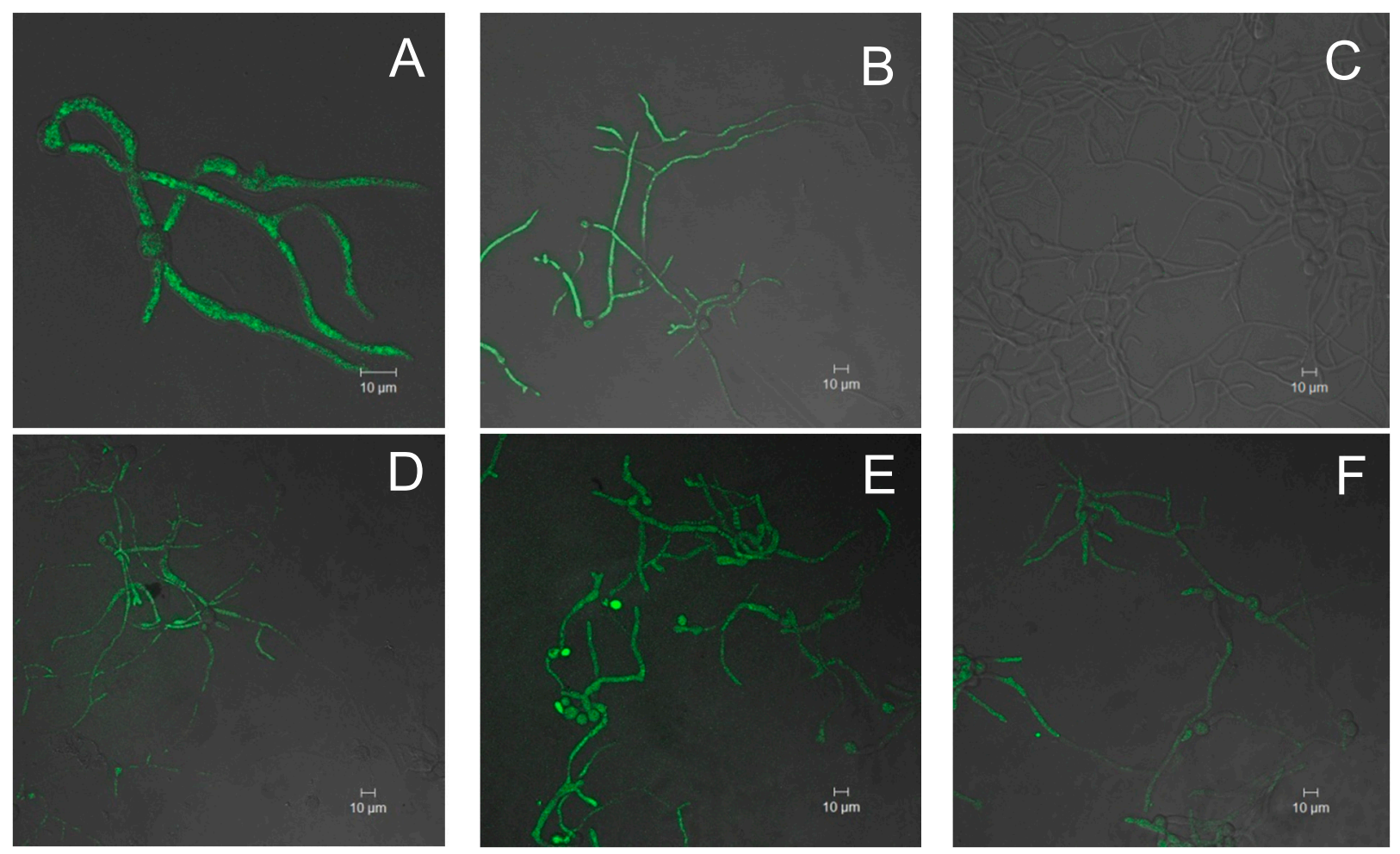

According to the results obtained from intracellular oxidation using the probe 2',7'-dichlorodihydrofluorescein diacetate (DCFH-DA) it was possible to observe that all tested extracts presented an accumulation of reactive oxygen species (ROS) at $40 \mathrm{ppm}$ demonstrating an oxidative stress, similar to that observed in the presence of farnesol (positive control). These results suggest that these extracts at the concentration tested, exerted a prooxidant effect on $B$. cinerea what would cause a toxic effect, inhibiting mycelial growth.

Therefore, based on the results obtained in this work, it is not possible to correlate antioxidant and/or prooxidant activity determined by cyclic voltammetry with $B$. cinerea mycelial growth inhibition. Probably, because multiple variables are involved in the in vivo assay as different mechanisms of action producing cell death by membrane damage, stress, etc. and the antioxidant cellular mechanisms which are not considered by the in vitro tests. 


\section{Experimental Section}

\subsection{Grape Pomaces}

Pomaces were obtained after grape fermentation from $V$. vinifera varieties (Cabernet Sauvignon, Carménère and Syrah) from the 2009 harvest season from Misiones de Rengo Vineyard (Rengo, Chile). Pomaces were maintained at $-20^{\circ} \mathrm{C}$ until they were used.

\subsection{General Extraction Process}

Extracts were prepared from whole and ground grape pomace and the phenolic compounds were extracted by three different systems:

(A) Methanol extraction: Whole and ground grape pomace (400 g) were extracted with methanol/ $\mathrm{HCl}(1 \% \mathrm{v} / \mathrm{v}, 1.5 \mathrm{~L})$ for $4 \mathrm{~h}$ with constant agitation at $4{ }^{\circ} \mathrm{C}$. Then extracts were concentrated in a rotary evaporator and distilled water $(20 \mathrm{~mL})$ was added and volume was decreased in a rotary evaporator. Water addition was repeated three times. Finally, aqueous suspension or crude extract was subjected to sequential liquid-liquid extraction with hexane, chloroform and finally ethyl acetate;

(B) Ethanol extraction: (i) System 1: Whole and ground grape pomace (400 g) were extracted with ethanol $(70 \% \mathrm{v} / \mathrm{v}, 1.5 \mathrm{~L})$ for $4 \mathrm{~h}$ with constant agitation at $4{ }^{\circ} \mathrm{C}$. Extracts were concentrated in a rotary evaporator to afford crude extracts which were subjected to sequential liquid-liquid extraction with hexane, chloroform and finally ethyl acetate; (ii) System 2: whole and ground grape pomace (400 g) were extracted with ethanol $(70 \% \mathrm{v} / \mathrm{v}, 1.5 \mathrm{~L})$ for $4 \mathrm{~h}$ with constant agitation at $4{ }^{\circ} \mathrm{C}$. Extracts were concentrated in a rotary evaporator and distilled water $(20 \mathrm{~mL})$ was added and then the volume was reduced on a rotary evaporator. Water addition was repeated three times. Finally, aqueous suspension or crude extract was subjected to sequential liquid-liquid extraction with hexane, chloroform and finally ethyl acetate;

(C) Soxhlet: whole grape pomace (10 g) was submitted to a Soxhlet extraction using methanol in a solid-liquid ratio of $1: 20$ at $40{ }^{\circ} \mathrm{C}$ for $12 \mathrm{~h}$. Methanol was partially removed under reduced pressure. This methanol extract was subjected to liquid-liquid extraction with hexane, giving a methanolic and a hexane phase.

\subsection{Determination of Total Phenol Content}

The amount of total phenol, in different extracts, was determined by Folin-Ciocalteu's reagent method. Total phenols content were expressed as gallic acid equivalent (milligrams per gram of extracted compounds) [35].

\subsection{Analysis of Phenolic Compounds in Different Extractions}

The HPLC analysis was performed as described by Mendoza et al. [36]. A Waters 600 HPLC chromatograph (Waters, Milford, MA, USA) equipped with a Waters 2990 diode array detector, and a Symmetry C-18 $(5 \mu \mathrm{m}, 3.9 \mathrm{~mm} \times 150 \mathrm{~mm})$ column (Waters) was used. The solvent system consisted of $1 \%$ aqueous formic acid (A) and $1 \%$ formic acid in acetonitrile (B). The initial composition of the mobile phase was $95 \% \mathrm{~A}$ and $5 \% \mathrm{~B}$. With linear gradients the composition changed to $75 \% \mathrm{~A}$ and 
25\% $\mathrm{B}$ within $45 \mathrm{~min}$, and 50\% $\mathrm{A}$ and $50 \% \mathrm{~B}$ within $60 \mathrm{~min}$. The flow rate was $0.8 \mathrm{~mL} / \mathrm{min}$. Identification of phenolic compounds was done by comparing their retention times and UV-Vis spectra with standards. Gallic acid, protocatechuic acid, vanillic acid, sinapic acid, syringic acid, ellagic acid, (+)-catechin, (-)-epicatechin, epigallocatechin, caffeic acid, vanillin, $p$-coumaric acid, 4-hydroxy-3,5-dimethoxybenzaldehyde, 4-hydroxyphenylacetic acid, transresveratrol, quercetin, kaempferol and myricetin were used as standards.

\subsection{Determination in Vitro of Antioxidant or Prooxidant Activity of Grape Pomace Extracts by Cyclic Voltammetry}

The antioxidant and prooxidant effects of extracts was determined using cyclic voltammetry as described by Simić et al. [27]. Electrochemical experiments were performed in a three-compartment glass cell, with a glassy carbon $\left(\mathrm{A}=0.071 \mathrm{~cm}^{2}\right)$ working electrode. Saturated $\mathrm{Ag} / \mathrm{AgCl} / \mathrm{KCl}$ was used as reference electrode with respect to which all the potentials are quoted, and a $\mathrm{Pt}$ coil $\left(\mathrm{A}=10 \mathrm{~cm}^{2}\right)$ was used as the counter electrode. The glassy carbon electrode was polished with $0.25 \mu \mathrm{m}$ alumina and ultrasonicated for $5 \mathrm{~min}$ before each experiment. Oxidant and prooxidant behavior was determined by potentiodynamically cycling the electrode between $-0.2 \mathrm{~V}$ and $2 \mathrm{~V}$ at a scan rate of $0.01 \mathrm{~V} / \mathrm{s}$ in dichloromethane containing $350 \mu \mathrm{g} / \mathrm{mL}$ of the extracts in the presence of $0.1 \mathrm{M}$ tetrabutylammonium perchlorate. The solution was purged with nitrogen (ultra-pure grade) during each measurement. All the experiments were carried out at room temperature in an inert atmosphere. Electrochemical measurements were performed using a CHI604C Bipotentiostat (CH Instruments, Austin, TX, USA) along with $\mathrm{CH}$ Instruments software.

\subsection{Determination of in Vivo Prooxidant Activity of Grape Pomace Extracts}

To determine the in vivo prooxidant activity of grape pomace extracts, the effect of the extracts on mycelial growth and on the production of oxidative stress in $B$. cinerea was evaluated.

\subsubsection{Fungal Isolate and Culture Conditions}

In this study $B$. cinerea strain G29 was used. This strain was originally isolated from naturally infected grapes ( $V$. vinifera) [37] and was maintained on malt-yeast extract agar slants $(2 \%(w / v)$ malt extract, $0.2 \%(w / v)$ yeast extract and $1.5 \%(w / v)$ agar) at $4{ }^{\circ} \mathrm{C}$. The fungus was grown in the dark on malt-yeast extract agar medium or soft agar medium $(2 \%(w / v)$ malt extract, $0.2 \%(w / v)$ yeast extract and $0.6 \%(w / v)$ agar $)$.

\subsubsection{Determination in Vivo Prooxidant Activity Using B. cinerea as Model Organism}

The antifungal activity on mycelial growth of $B$. cinerea of different extracts and phenolic compounds was assessed using a protocol described by Mendoza et al. [9]. All the experiments were performed at least in triplicate and with adequate controls (solvent and positive controls). Additionally, a commercial fungicide was used as positive control. Antifungal effect results were expressed as $\mathrm{IC}_{50}$ (concentration that reduced mycelial growth by $50 \%$ ) determined by regressing the inhibition of radial growth values (percent control) at different extract or compound concentration after $48 \mathrm{~h}$ of incubation. 
To demonstrate in vivo prooxidant activity of some grape pomace extracts the probe 2,7-dichlorodihydrofluorescein diacetate (DCFH-DA) in protoplasts of $B$. cinerea treated with $40 \mathrm{ppm}$ of extracts for $6 \mathrm{~h}$ was used according to the manufacturer's instructions. The protoplasts were obtained according the protocols described by Cotoras et al. [33]. After $15 \mathrm{~min}$ of incubation with the probe, the protoplasts attached to the coverslips were washed three times with minimal medium and mounted on slides according to describe by Cotoras et al. [33]. For mounting the samples DABCO (1,4-diazabicyclo[2.2.2] octane, $10 \mu \mathrm{L})$ was used. Finally, the protoplasts of $B$. cinerea were visualized under a confocal microscope (LSM 510, Carl Zeiss, Göttingen, Germany) at an excitation wavelength of $488 \mathrm{~nm}$ and an emission wavelength of $540 \mathrm{~nm}$. Hyphae without exposure to oxidative stress were used as negative control; as for the positive control hyphae exposed to hydrogen peroxide (300 $\mathrm{mM})$ or farnesol (100 mM) according Joo and Jetten [34] was used. Each experiment was performed in duplicate.

\section{Conclusions}

Depending on the method of extraction of the grape residues and the grape variety (Cabernet Sauvignon, Carménère and Syrah), the extracts show antioxidant and/or prooxidant activity. However, no correlation can be established between the anodic oxidation potentials of the extracts and their effect on the fungus $B$. cinerea.

\section{Acknowledgments}

This study was partially supported by FONDECYT 1130389 and FONDEF CA12i10054 Grants.

\section{Author Contributions}

M.C. and L.M. participated in the experiment designs and in the result analyses. E.S. have written and revised the paper. R.M. performed the determination of the phenolic compound profiles. H.V. prepared the extracts, performed the biological and voltammetry assays. M.A. participated in the analysis of the cyclic voltagrams.

\section{Conflicts of Interest}

The authors declare no conflict of interest.

\section{References}

1. Ruggieri, L.; Cadena, E.; Martinez-Blanco, J.; Gasol, C.M.; Rieradevall, J.; Gabarrel, X.; Gea, T.; Sort, X.; Sánchez, A. Recovery of organic wastesin the Spanish wine industry: Technical, economic and environmental analyses of the composting process. J. Clean. Prod. 2009, 17, 830-838.

2. De Campos, L.M.; Leimann, F.; Pedrosa, R.; Ferreira, S. Free radical scavenging of grape pomace extracts from Cabernet Sauvingnon (Vitis vinifera). Bioresour. Technol. 2008, 99, 8413-8420.

3. Schieber, A.; Stintzing, F.C.; Carle, R. By-products of plant food processing as a source of functional compounds recent developments. Trends Food Sci. Technol. 2001, 12, 401-413. 
4. Xia, E.Q.; Deng, G.F.; Guo, Y.J.; Li, H.B. Biological activities of polyphenols from grapes. Int. J. Mol. Sci. 2010, 11, 622-646.

5. Rasmussen, S.E.; Frederiksen, H.; Struntze, K.; Poulsen, L. Dietary proanthocyanidins: occurrence, dietary intake, bioavailability, and protection against cardiovascular disease. Mol. Nutr. Food Res. 2005, 49, 159-174.

6. Arts, I.C.; Hollman, P.C. Polyphenols and disease risk in epidemiologic studies. Am. J. Clin. Nutr. 2005, 81, 317S-325S.

7. Cole, G.M.; Lim, G.P.; Yang, F.; Teter, B.; Begum, A.; Ma, Q.; Harris-White, M.E.; Frautschy, S.A. Prevention of Alzheimer's disease: Omega-3 fatty and phenolic anti-oxidant interventions. Neurobiol. Aging 2006, 26 (Suppl. S1), 133-136.

8. Hertog, M.G.; Feskens, E.J.; Hollman, P.C.; Katan, M.B.; Kromhout, D. Dietary flavonoids and cancer risk in the Zutphen Elderly Study. Nutr. Cancer 1994, 22, 175-184.

9. Mendoza, L.; Yañez, K.; Vivanco, M.; Melo, R.; Cotoras, M. Characterization of extracts from winery by-products with antifungal activity against Botrytis cinerea. Ind. Crop Prod. 2013, 43, 360-364.

10. Jarvis, WR. Epidemiology in the Biology of Botrytis; Coley-Smith, J.R., Verhoeff, K., Jarvis, W.R., Eds.; Academic Press: London, UK, 1980; pp. 219-250.

11. Rice-Evans, C.A.; Miller, N.J.; Bolwell, P.G.; Bramley, P.M.; Pridham, J.B. The relative antioxidant activities of plant-derived polyphenolic flavonoids. Free Radic. Res. 1995, 22, 375-383.

12. Rice-Evans, C.A.; Miller, N.J.; Paganga, G. Structure-antioxidant activity relationships of flavonoids and phenolic acids. Free Radic. Biol. Med. 1996, 20, 933-956.

13. Hagerman, A.E.; Riedl, K.M.; Jones, G.A.; Sovik, K.N.; Ritchard, N.T.; Hartzfeld, P.W.; Riechel, T.L. High molecular weight plant polyphenolics (tannins) as biological antioxidants. J. Agric. Food Chem. 1998, 46, 1887-1892.

14. Fukumoto, L.R.; Mazza, G. Assessing antioxidant and prooxidant activities of phenolic compounds. J. Agric. Food Chem. 2000, 48, 3597-3604.

15. Sharma, M.; Mnoharlal, R.; Puri, N.; Prasad, R. Antifungal curcumin induces reactive oxygen species and triggers an early apoptosis but prevents hyphae development by targeting the global repressor TUP1 in Candida albicans. Biosci. Rep. 2010, 30, 391-404, doi:10.1042/BSR20090151.

16. Lupetti, A.; Danesi, R.; Del, C.M.M.; Kelly, S. Molecular basis of resistance to azole antifungals. Trends Mol. Med. 2002, 8, 76-81.

17. Latifa, B.; Bouchra, L.; Sanaa, A; Najat, C.; Fouzia, C.; Adnane, R. Comparative study of the antifungal activity of some essential oils and their major phenolic components against Aspergillus niger using three different methods. Afr. J. Biotechnol. 2012, 11, 14083-14087.

18. Cotoras, M.; Mendoza, L.; Muñoz, A.; Yañez, K.; Castro, P.; Aguirre, M. Fungitoxicity against Botrytis cinerea of a flavonoid isolated from Pseudognaphalium robustum. Molecules 2011, 16, 3885-3895.

19. Castañeda-Ovando, A.; Pacheco-Hernández, M.D.L.; Páez-Hernández, M.E.; Rodríguez, J.A.; Galán-Vidal, C.A. Chemical studies of anthocyanins: A review. Food Chem. 2009, 113, 859-871.

20. Yilmaz, Y.; Toledo, R.T. Major flavonoids in grape seeds and skins: Antioxidant capacity of catechin, epicatechin, and gallic acid. J. Agric. Food Chem. 2004, 52, 255-260. 
21. Li, X.; Wang, X.; Chen, D.; Chen, S. Antioxidant activity and mechanism of protocatechuic acid in vitro. Funct. Foods Health Dis. 2011, 7, 232-244.

22. Andersen, M.L.; Lauridsen, R.K.; Skibsted, L.H. Optimizing the use of phenolic compounds in foods. In Phytochemical Functional Foods; Johnson, I., Williamson, G., Eds.; Woodhead Publishing Ltd.: Cambridge, UK, 2003; pp. 315-346.

23. Moure, A.; Cruz, J.M.; Franco, D.; Domínguez, J.M.; Sineiro, J.; Dominguez, H.; Núñez, M.J.; Parajó, J.C. Natural antioxidants from residual sources. Food Chem. 2001, 72, 145-171.

24. Schwartz, K.; Bertelsen, G.; Nissen, L.R.; Gardner, P.T.; Heinonen, M.I.; Hopia, A.; Huynh-Ba, T.; Lambelet, P.; McPhail, D.; Skibsted, L.H.; et al. Investigation of plant extracts for the protection of processed foods against lipid oxidation. Comparison of antioxidant assays based on radical scavenging, lipid oxidation and analysis of the principal antioxidant compounds. Eur. Food Res. Technol. 2001, 212, 319-328.

25. Sakihama, Y.; Cohen, M.F.; Grace, S.C.; Yamasaki, H. Plant phenolic antioxidant and prooxidant activities: Phenolics-induced oxidative damage mediated by metals in plants. Toxicology 2002, 177, 67-80.

26. Samra, M.A.; Chedea, V.S.; Economou, A.; Calokerinos, A.; Kefalas, P. Antioxidant/prooxidant properties of model phenolic compounds: Part I. Studies on equimolar mixtures by chemiluminescence and cyclic voltammetry. Food Chem. 2011, 125, 622-629.

27. Simić, A.; Manojlović, D.; Šegan, D.; Todorović, M. Electrochemical behavior and antioxidant and prooxidant activity of natural phenolics. Molecules 2007, 12, 2327-2340.

28. Balasundram, N.; Sundaram, K.; Samman, S. Phenolic compounds in plants and agri-industrial by-products: Antioxidant activity, occurrence, and potential uses. Food Chem. 2006, 99, 191-203.

29. Middleton, J.; Kandaswami, C.; Theoharides, T.C. The effects of plant flavonoids on mammalian cells: Implications for inflammation. Heart Dis. Cancer Pharmacol. Rev. 2000, 52, 673-751.

30. Lambert, J.; Elias, R. The antioxidant and pro-oxidant activities of green tea polyphenols: A role in cancer prevention. Arch. Biochem. Biophys. 2010, 501, 65-72.

31. Gunckel, S.; Santander, P.; Cordano, G.; Ferreira, J.; Munoz, S.; Nunez-Vergara, L.; Squella, J.A. Antioxidant activity of gallates: An electrochemical study in aqueous media. Chem. Biol. Interact. 1998, 114, 45-49.

32. Gomes, A.; Fernandes, E.; Lima, J. Fluorescence probes used for detection of reactive oxygen species. J. Biochem. Biophys. Methods 2005, 65, 45-48.

33. Cotoras, M.; Castro, P.; Vivanco, H.; Melo, R.; Mendoza, L. Farnesol induces apoptosis-like phenotype in the phytopathogenic fungus Botrytis cinerea. Mycologia 2013, 105, 28-33.

34. Joo, J.H.; Jetten, A.M. Molecular mechanisms involved in farnesol-induced apoptosis. Cancer Lett. 2009, 287, 123-135.

35. McDonald, S.; Prenzler, P.; Autolovich, M.; Robards, K. Phenolic content and antioxidant activity of olive extracts. Food Chem. 2001, 73, 73-84.

36. Mendoza, L.; Cotoras, M.; Vivanco, M.; Matsuhiro, B.; Torres, S.; Aguirre, M. Evaluation of antifungal properties against the phytopathogeic fungus Botrytis cinerea of anthocyanin rich-extracts obtained from grape pomaces. J. Chil. Chem. Soc. 2013, 58, 1725-1727. 
37. Munoz, G.; Hinrichsen, P.; Brygoo, Y.; Giraud, T. Genetic characterization of Botrytis cinerea populations in Chile. Mycol. Res. 2002, 106, 594-601.

Sample Availability: Samples of the compounds are available from the authors.

(C) 2014 by the authors; licensee MDPI, Basel, Switzerland. This article is an open access article distributed under the terms and conditions of the Creative Commons Attribution license (http://creativecommons.org/licenses/by/4.0/). 